\title{
Puerarin inhibits bladder cancer cell proliferation through the mTOR/p70S6K signaling pathway
}

\author{
KEHUA JIANG $^{1,2^{*}}$, HONGBO CHEN $^{2 *}, \mathrm{KUN} \mathrm{TANG}^{1}$, WEI GUAN ${ }^{1}$, HUI ZHOU $^{1}$, \\ XIAOLIN GUO $^{1}$, ZHIQIANG CHEN ${ }^{1}$, ZHANGQUN YE ${ }^{1}$ and HUA XU ${ }^{1}$ \\ ${ }^{1}$ Department of Urology, Tongji Hospital, Tongji Medical College, Huazhong University of \\ Science and Technology, Wuhan, Hubei 430030; ${ }^{2}$ Department of Urology, The Central Hospital of \\ Enshi Tujia and Miao Autonomous Prefecture, Enshi, Hubei 445000, P.R. China
}

Received March 23, 2016; Accepted September 22, 2017

DOI: $10.3892 / \mathrm{ol} .2017 .7298$

\begin{abstract}
Puerarin, as a novel oncotherapeutic agent, may exert anticancer effects and inhibit the proliferation of cancer cells. To explore the effects of puerarin on human bladder cancer cells, and to elucidate the potential mechanism underlying these effects, a Cell Counting Kit- 8 assay was used to examine the proliferation of T24 and EJ cells following puerarin treatment. The effects of puerarin treatment on the cell cycle were detected by flow cytometry (FCM), while puerarin-induced cell apoptosis was detected by terminal deoxynucleotidyl transferase dUTP nick end labeling and FCM, and the cellular ultrastructural morphological changes were observed by transmission electron microscopy. Cell invasion was examined using a Transwell assay with Matrigel. The expression levels of mechanistic target of rapamycin (mTOR), phosphorylated (p)-mTOR, p70-S6 kinase (p70S6K) and p-p70S6K proteins in the mTOR signaling pathway were then assessed by western blotting. The results demonstrated that puerarin may inhibit bladder cancer cell viability, block the cell cycle in the G0/G1 phase and induce apoptosis in bladder cancer cells. The expression levels of p-mTOR and p-p70S6K proteins were downregulated, while no change was observed in the expression levels of mTOR and p70S6K proteins when T-24 and EJ cells were treated by puerarin. In the present study, puerarin was demonstrated to inhibit the viability of human bladder cancer cells. These effects may be due to the puerarin-induced downregulation of proteins in the
\end{abstract}

Correspondence to: Professor Zhiqiang Chen or Professor Hua Xu, Department of Urology, Tongji Hospital, Tongji Medical College, Huazhong University of Science and Technology, 1095 Jiefang Avenue, Wuhan, Hubei 430030, P.R. China

E-mail: zhqchen8366@163.com

E-mail: xuhuawhu@163.com

*Contributed equally

Key words: puerarin, bladder cancer, mechanistic target of rapamycin signaling pathway, apoptosis, p70S6K
mTOR/p70S6K signaling pathway, and the present study may provide the experimental basis for puerarin to be considered as a promising novel anti-tumor drug for the treatment of bladder cancer.

\section{Introduction}

Bladder cancer is increasingly common globally and its morbidity and mortality rates are the fourth and seventh highest in men as estimated by the American Cancer Society in 2015, respectively (1). Therefore, bladder cancer is a major burden to public health. In total, $\sim 75 \%$ of newly diagnosed bladder cancer cases are non-muscle-invasive bladder cancer (NMIBC) $(2,3)$. The recommended treatment for patients with NMIBC is transurethral resection of bladder tumor. However, bladder cancers have a high recurrence rate, and $25 \%$ of patients with NMIBC develop into muscle-invasive bladder cancer following treatment $(4,5)$. Although numerous chemotherapeutic drugs have been demonstrated to inhibit tumor recurrence and progression, their toxic side effects and chemosensitivity reduce the overall therapeutic effect for patients with NMIBC (6-9). Therefore, the identification of novel adjuvants or alternative agents for patients with NMIBC is urgently required. Previous studies have revealed that certain plants and microorganisms have anticancer effects, often characterized by low toxicity and few side-effects $(10,11)$.

Puerarin is the main isoflavone glycoside isolated from the traditional Chinese herb Radix pueraria lobate (12). Puerarin has been widely used as an antidiuretic, antipyretic and diaphoretic due to its various medicinal properties (12). Previous studies have demonstrated that puerarin may be used to treat neurodegenerative disorders $(13,14)$ and cardio-cerebrovascular disease $(15,16)$. In addition, puerarin may inhibit the apoptosis of human osteoblasts through the extracellular signal-regulated kinase signaling pathway (17). Puerarin may also exert anticancer effects and inhibit the growth of esophageal cancer cells, and this effect is associated with the mitochondrial pathway (18). It also inhibits proliferation and induces apoptosis in glioblastoma (19), gastric cancer (20) and colon cancer (21) cell lines. However, the effect of puerarin on human bladder cancer are unclear, and the underlying mechanisms remain elusive. Therefore, the present study investigated 
the anticancer effects and potential mechanisms underlying the effect of puerarin on human bladder cancer.

\section{Materials and methods}

Cell culture and reagents. Human bladder cancer T24 cell line and its derivative, the EJ cell line, were purchased from the China Center for Type Culture Collection (Wuhan University, Wuhan, China) (22). The cells were maintained in RPMI-1640 medium (Gibco; Thermo Fisher Scientific, Inc., Waltham, MA, USA). Puerarin was purchased from Shandong Fangming Pharmaceutical Group Co., Ltd. (Heze, China; injection grade; Chinese FDA approval no. H20033292). Dimethyl sulfoxide was purchased from Sigma-Aldrich; Merck KGaA (Darmstadt, Germany). Fetal bovine serum (FBS) was obtained from Gibco; Thermo Fisher Scientific, Inc. The bladder cancer T24 and EJ cell lines were cultured in RPMI-1640 medium with $10 \% \mathrm{FBS}$ and maintained at $37^{\circ} \mathrm{C}$ in a humidified atmosphere of $5 \% \mathrm{CO}_{2}$. The medium was changed every 2-3 days, and cells were subcultured until they reached $90 \%$ confluency prior to being harvested using trypsin.

Cell viability assay with Cell Counting Kit-8 (CCK-8). CCK-8 (Dojindo Molecular Technologies, Inc., Kumamoto, Japan) was utilized to quantify T24 and EJ cell viability. Cells were seeded onto 96-well plates at a density of $1 \times 10^{5}$ cells/well for $24 \mathrm{~h}$, and then incubated with RPMI-1640 medium containing various dilutions of puerarin $(0.01,0.1,1,10$ and $100 \mu \mathrm{mol} / \mathrm{l})$ and negative control (completed untreated) at $37^{\circ} \mathrm{C}$ in a $5 \% \mathrm{CO}_{2}$ humidified atmosphere for 24, 48 and $72 \mathrm{~h}$. Following incubation for the indicated times, $10 \mu \mathrm{l} \mathrm{CCK}-8$ solution was added to each well and incubated for $2 \mathrm{~h}$ at $37^{\circ} \mathrm{C}$ to examine the effect of puerarin on bladder cancer cell proliferation. Colorimetric analysis was performed at a wavelength of $490 \mathrm{~nm}$. Three independent experiments were performed in triplicate.

Transwell cell invasion assays. T24 and EJ cells were seeded in 12 -well culture plate at a density of $4 \times 10^{5}$ cells/well and incubated with puerarin $(100 \mu \mathrm{mol} / 1)$ at $37^{\circ} \mathrm{C}$ in a $5 \% \mathrm{CO}_{2}$ humidified atmosphere for 24,48 and $72 \mathrm{~h}$, with completely untreated cells used as the negative control group. The cells were then suspended in serum free RPMI-1640 medium and plated at a density of $2 \times 10^{5}$ cells/well in the upper chamber of Transwell plates with polycarbonate membranes (pore size, $8 \mu \mathrm{m}$ ) and diluted Matrigel coating (BD Biosciences, Franklin Lakes, NJ, USA). Complete medium (10\% FBS RMPI-1640; $600 \mu \mathrm{l}$ ) was added to the lower chamber. Following incubation for $18 \mathrm{~h}$ at $37^{\circ} \mathrm{C}$ in a $5 \% \mathrm{CO}_{2}$ humidified atmosphere, the cells that passed through the filters into the bottom wells were fixed in $100 \%$ methanol for $30 \mathrm{~min}$ at $4^{\circ} \mathrm{C}$ and stained with $0.5 \%$ crystal violet for $15 \mathrm{~min}$ at $37^{\circ} \mathrm{C}$. The number of cells in 10 randomly selected fields (magnification, x100) from each well was counted under an optical microscope (CX21; Olympus Corporation, Tokyo, Japan). The invasion assays were repeated at least three times.

Transmission electron microscopy. To observe the morphological changes of bladder cancer cell lines induced by puerarin with different time and concentration, T24 and EJ cells were pretreated with puerarin $(100 \mu \mathrm{mol} / \mathrm{l})$ for $0,24,48$ and $72 \mathrm{~h}$ at $37^{\circ} \mathrm{C}$, or were completely untreated in the negative control group. Additionally, T24 cells were treated with different concentrations of puerarin $(0,1,10$ and $100 \mu \mathrm{mol} / \mathrm{l})$ for $72 \mathrm{~h}$ at $37^{\circ} \mathrm{C}$. The cells were then collected with $447.2 \mathrm{x}$ g centrifugation for $5 \mathrm{~min}$ at room temperature and fixed with $2.5 \%$ glutaraldehyde for $2 \mathrm{~h}$ at $4^{\circ} \mathrm{C}$. Then the sample was treated with $1 \%$ osmium tetroxide for $30 \mathrm{~min}$ at $4^{\circ} \mathrm{C}$ and dehydrated in increasing concentrations of acetone (50, 70, 90 and 100\%; cat no. PYG0013; Boster Biological Technology, Pleasanton, CA, USA) at room temperature. The sample was embedded in embedding resin (cat no. 18109; Epon 812 embedding kit; Ted Pella, Inc., CA, USA) for $24 \mathrm{~h}$ at $60^{\circ} \mathrm{C}$, and a $50 \mathrm{~mm}$ ultrathin section was prepared with a microtome. The ultrastructure of cells was detected by transmission electron microscopy (Tecnai G2, FEI; Thermo Fisher Scientific, Inc., Waltham, MA, USA) at X100 magnification.

Cell cycle and apoptosis assay by flow cytometry (FCM). T24 cells were seeded in 4 - wells plates at a density of $4 \times 10^{5}$ cells/well and incubated with puerarin $(100 \mu \mathrm{mol} / \mathrm{l})$ at $37^{\circ} \mathrm{C}$ in a $5 \% \mathrm{CO}_{2}$ humidified atmosphere for $0,24,48$ and $72 \mathrm{~h}$, and control cells being completely untreated. The cells were then collected by trypsin and centrifugation. A total of $500 \mu \mathrm{l}$ binding buffer (including precooled $70 \%$ ethanol and $0.5 \mathrm{mmol} / \mathrm{l}$ EDTA) was added to each tube and incubated overnight in a $4^{\circ} \mathrm{C}$ refrigerator. Cells were then resuspended, centrifuged at a speed of $447.2 \mathrm{x} \mathrm{g}$ for $5 \mathrm{~min}$ at room temperature and washed twice with PBS. PBS containing $0.1 \%$ Triton X-100 and $50 \mu \mathrm{g} / \mathrm{ml}$ RNAse was applied to the resuspended cells. Subsequently, cells were incubated in $90 \mu 1$ propidium iodide (PI) buffer (Sigma-Aldrich; Merck KGaA) for $30 \mathrm{~min}$ at room temperature in the dark, and cell cycle analyses were performed by FCM within $1 \mathrm{~h}$. Cells were collected and subjected to Annexin V and PI staining using an Annexin V-fluorescein isothiocyanate apoptosis detection kit (Vazyme, Piscataway, NJ, USA), following the manufacturer's protocol. Apoptotic cells were then detected by FCM (BD FACSCalibur; BD Biosciences).

Cell apoptosis detection by terminal deoxynucleotidyl transferase dUTP nick end labeling (TUNEL) staining. T24 and EJ cells with $4 \times 10^{5} /$ well cell density were pretreated with puerarin $(0$ and $100 \mu \mathrm{mol} / \mathrm{l})$ and incubated at $37^{\circ} \mathrm{C}$ in a $5 \%$ $\mathrm{CO}_{2}$ humidified atmosphere for 24,48 and $72 \mathrm{~h}$, with negative control cells being completely untreated. Cell apoptosis was analyzed by TUNEL assay, according to the manufacturer's protocol (TUNEL apoptosis assay kit; Roche Diagnostics $\mathrm{GmbH}$, Mannheim, Germany). Apoptosis of cells was analyzed by counting the positive cells, as well as the total number of cells, at 10 randomly selected fields at x400 magnification in a blinded manner using a fluorescence microscope (IX71; Olympus Corporation).

Western blot analysis. To observe the effect of puerarin on the expression level of protein in bladder cancer lines by different time and concentration, T24 cells, following treatment with puerarin $(0$ and $100 \mu \mathrm{mol} / \mathrm{l})$, were incubated for $0,24,48$ and $72 \mathrm{~h}$, respectively. Additionally, T24 and EJ cells were treated with different concentrations of puerarin $(0,1$ and $100 \mu \mathrm{mol} / \mathrm{l})$ for $72 \mathrm{~h}$, respectively. Cells were then collected using trypsin 
and were centrifuged at a speed of $12,745.2 \mathrm{xg}$ for $15 \mathrm{~min}$ at $4{ }^{\circ} \mathrm{C}$, and lysed for $30 \mathrm{~min}$ at $4^{\circ} \mathrm{C}$ by lysis buffer containing $50 \mathrm{mM}$ Tris (pH 7.4), $10 \%$ glycerol, $50 \mathrm{mM} \mathrm{NaCl}, 1 \mathrm{mM}$ EDTA and $1 \%$ Triton X-100. All protein extraction processes were performed according to the product specification. A bicinchoninic protein assay kit (Beyotime Institute of Biotechnology, Haimen, China) was used to measure the protein concentration. Equal amounts of protein $(50 \mu \mathrm{g})$ were then separated by electrophoresis on $12 \%$ SDS-PAGE and proteins were transferred to polyvinylidene fluoride membranes. The membranes were blocked in 5\% non-fat milk solution for $2-4 \mathrm{~h}$ at $4^{\circ} \mathrm{C}$ in a freezer and washed twice with TBST solution (0.1\% Tween-20). Membranes were incubated overnight at $4^{\circ} \mathrm{C}$ with the following antibodies: Polyclonal rabbit mammalian target of rapamycin (mTOR; cat no. A00003; 1:1,000, Boster Biological Technology, Pleasanton, CA, USA); polyclonal rabbit phosphorylated (p-) mTOR (cat no. 20301782-1; 1:2,000; Bioworld Technology, Inc., St. Louis Park, MN, USA); polyclonal rabbit P70-S6 kinase 1 (p70S6K; cat no. 20313303-1; 1:2,000; Bioworld Technology, Inc.); and polyclonal rabbit p-p70S6K (cat no. 20314827-1; 1:2,000; Bioworld Technology, Inc.); $\beta$-actin (cat no. BM0626; 1:1,000; Boster Biological Technology). Membranes were then washed three times with TBST solution, incubated with horseradish peroxidase-conjugated secondary antibodies (goat anti-mouse immunoglobulin G; cat no. BA1051 and cat no. BA1055; 1:800; Boster Biological Technology) for $2 \mathrm{~h}$ at $4^{\circ} \mathrm{C}$ freezer, and the expression of proteins was then detected with an enhanced chemiluminescence kit (super-sensitive ECL ready-to-use substance kit; cat no. AR1173; Boster Biological Technology) with GeneSys imaging software (Gene Gnome, version: 1.5.9.0; Syngene, Frederick, MD, USA).

Statistical analysis. SPSS software (version 16; SPSS, Inc., Chicago, IL, USA) was used for statistical analysis. All data were calculated as the mean \pm standard deviation. One-way ANOVA and the Student-Neuman-Keuls method were utilized to analyze the results between treated and control groups, and a Student's t test was used to compare two groups. $\mathrm{P}<0.05$ was considered to indicate a statistically significant difference.

\section{Results}

Puerarin inhibits the viability of bladder cancer cells. Human bladder T24 and EJ cells were pretreated with puerarin $(0,0.01$, $0.1,1,10$ and $100 \mu \mathrm{mol} / \mathrm{l})$ for 24,48 and $72 \mathrm{~h}$. Subsequently, cell viability was measured using the CCK- 8 assay, and cell viability was revealed to be inhibited in a concentration- and time-dependent manner by puerarin treatment. As presented in Fig. 1, the relative viability of T24 and EJ cells following $100 \mu \mathrm{mol} / 1$ puerarin treatment for $72 \mathrm{~h}$ was 47 and $50 \%$, respectively, compared with the untreated control cells. With increased incubation time, the relative cell viability of T24 and EJ cells treated with puerarin decreased compared with the untreated control cells. Therefore, a dosage of $100 \mu \mathrm{mol} / 1$ puerarin and incubation periods of 24,48 and $72 \mathrm{~h}$ were used for subsequent experiments.

Puerarin inhibits the invasion of bladder cancer cells. Following pretreatment of human bladder cancer T24 and EJ cells with 0 or $100 \mu \mathrm{mol} / 1$ puerarin for 24,48 and $72 \mathrm{~h}$, cell
A

T24

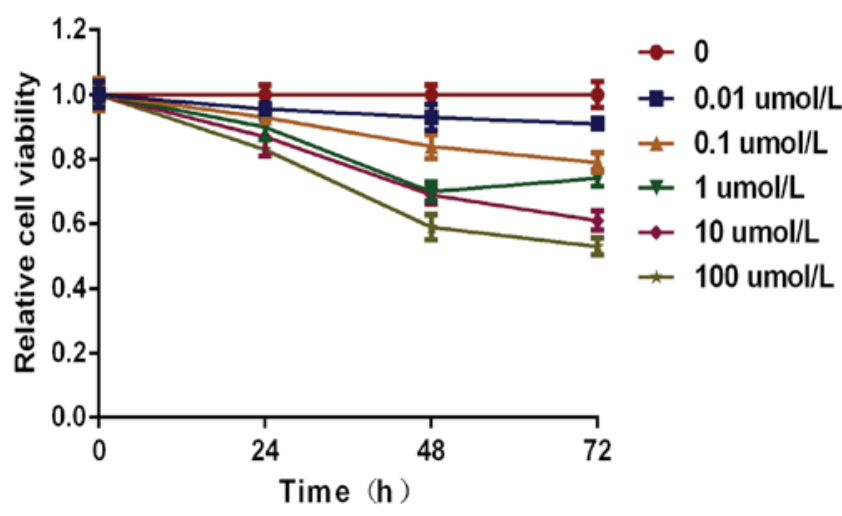

B

EJ

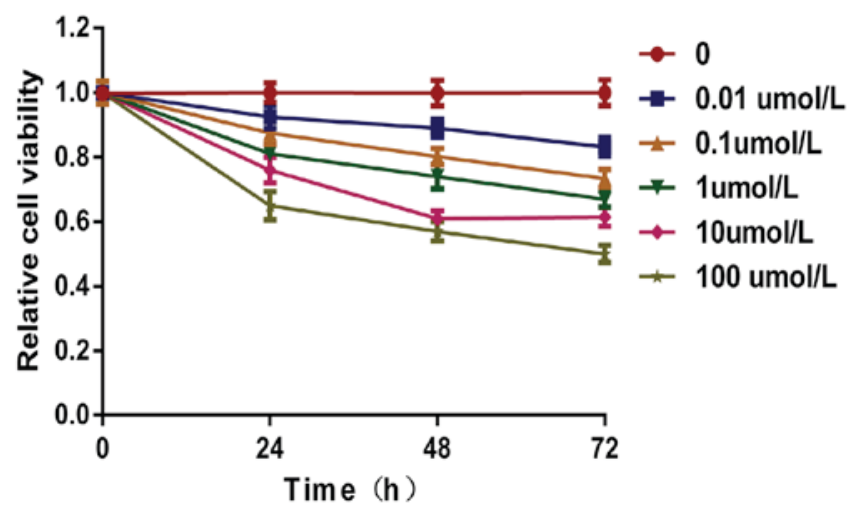

Figure 1. Puerarin inhibited bladder cancer T24 and EJ cell viability. Effects of puerarin on the viability of (A) T24 and (B) EJ cells. Values are given as a percentage of untreated control cells. The data are presented as the average of triplicate results from a representative experiment. Bars represent the standard deviation.

invasion was measured by Transwell assay. As presented in Fig. 2, puerarin treatment significantly inhibited the invasion of bladder cancer T24 and EJ cells, compared with the control group $(\mathrm{P}<0.05)$.

Morphological changes in bladder cancer cells are induced by puerarin treatment. Transmission electron microscopy was used to examine the morphological changes induced by puerarin. As presented in Fig. 3, the untreated control groups contained cells with intact nuclear membranes, large and circular nuclei, increased chromatin, abundant mitochondria and endoplasmic reticulum with normal morphology. However, for T24 and EJ cells treated with puerarin for $24 \mathrm{~h}$, lumped chromatin accumulation was observed inside the nuclear membrane, the mitochondria were impaired due to pyknosis, resulting in membrane ruptures and fewer mitochondria overall in the puerarin-treated group, and a large number of autophagocytic vacuoles were formed. Compared with puerarin treatment for $24 \mathrm{~h}$, when the T24 and EJ cells were incubated with puerarin for 48 and $72 \mathrm{~h}$, the cells became smaller, an increased number of organelles were lost, the nuclear membranes were partially disrupted and the nuclei were broken up (Fig. 3). The T24 cells treated with different concentrations of puerarin $(1,10$ and $100 \mu \mathrm{mol} / \mathrm{l})$ for $72 \mathrm{~h}$ exhibited the same inhibitory effect (Fig. 3). 
A T24

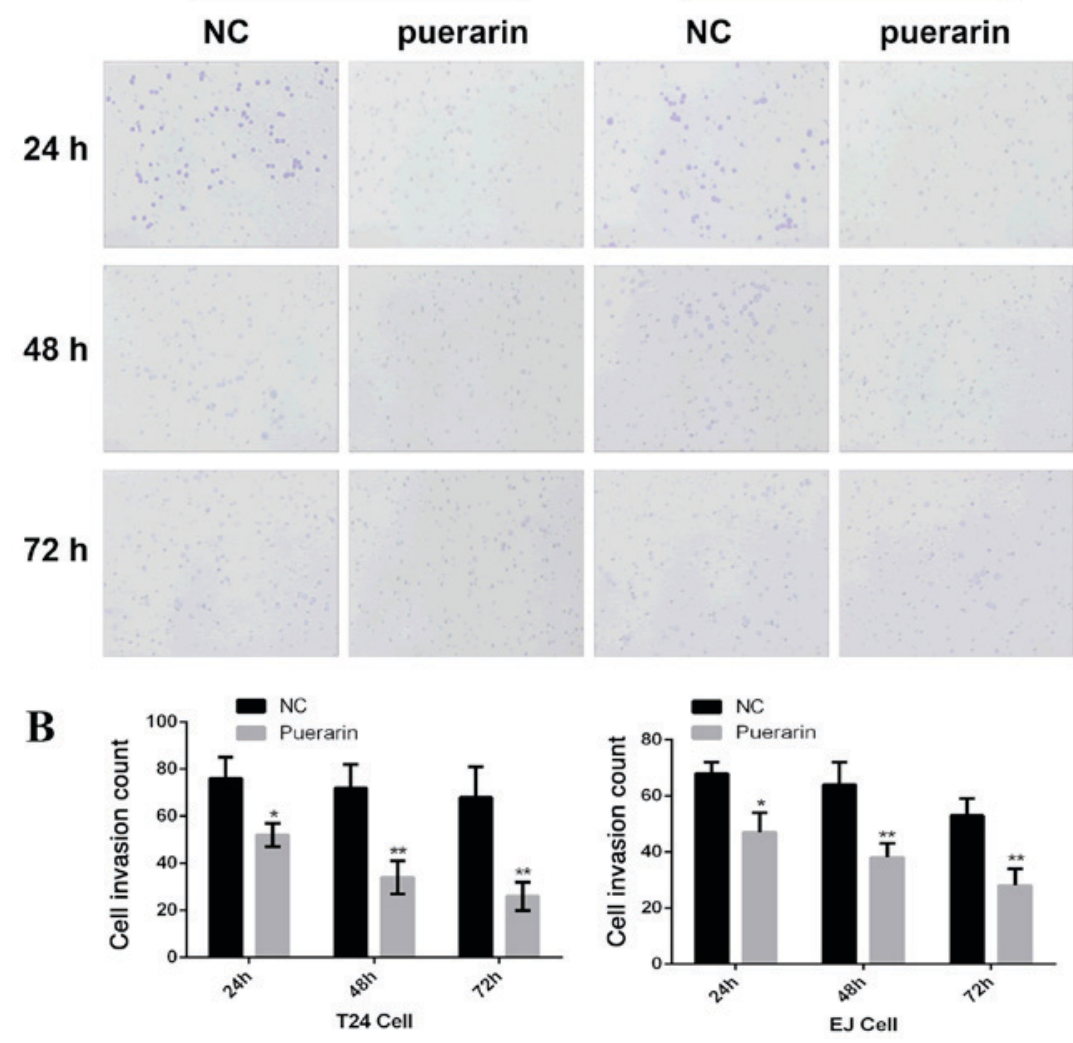

Figure 2. Puerarin inhibited the invasion of bladder cancer cells. (A) T24 and EJ cells treated with puerarin (100 $\mu$ mol/l) for 24,48 and $72 \mathrm{~h}$, and then harvested. Invasion was analyzed by Transwell assay with Matrigel; magnification, x100. (B) The quantified number of invaded cells. The data are presented as the average of triplicate results from a representative experiment. Bars, standard deviation. ${ }^{*} \mathrm{P}<0.05,{ }^{* *} \mathrm{P}<0.01$ vs. negative control.

Puerarin affects the cell cycle and apoptosis of bladder cancer cells. FCM was applied to detect alterations in the cell cycle distribution induced by puerarin treatment in the T24 cell line. As presented in Fig. 4, compared with the $0 \mathrm{~h}$ (completed untreated) control group, cultivating T24 cells with $100 \mu \mathrm{mol} / \mathrm{l}$ puerarin for 24,48 and $72 \mathrm{~h}$ resulted in $10.5,12.8$ and $17.5 \%$ increases in the percentage of cells in the G0/G1 phase, respectively. This was accompanied by a decrease in the percentage of cells in the $\mathrm{G} 2 / \mathrm{M}$ phase, but no significant difference was observed in the percentage of cells in the $S$ phrase, which indicated that puerarin induces cell cycle arrest at the G0/G1 phase in bladder cancer T24 cells. The results also revealed that the apoptotic rate of T24 cells increased following treatment with puerarin for 24, 48 and $72 \mathrm{~h}$ (Fig. 4).

Apoptotic effect of puerarin in bladder cancer cells. A TUNEL assay was performed to detect the apoptosis rate of bladder cancer T24 and EJ cells treated with puerarin. Compared with the control group, the results demonstrated the apoptosis of T24 and EJ cells was increased following treatment with puerarin for 24, 48 and $72 \mathrm{~h}$ (Fig. 5).

Antitumor effects of puerarin are mediated by the mTOR signaling pathway. The mTOR signaling pathway is involved in the tumorigenesis, development and prognosis of bladder urothelium carcinoma, and is essential for the regulation of autophagy $(23,24)$. Therefore, to investigate the mechanisms underlying puerarin- induced cell apoptosis, western blotting

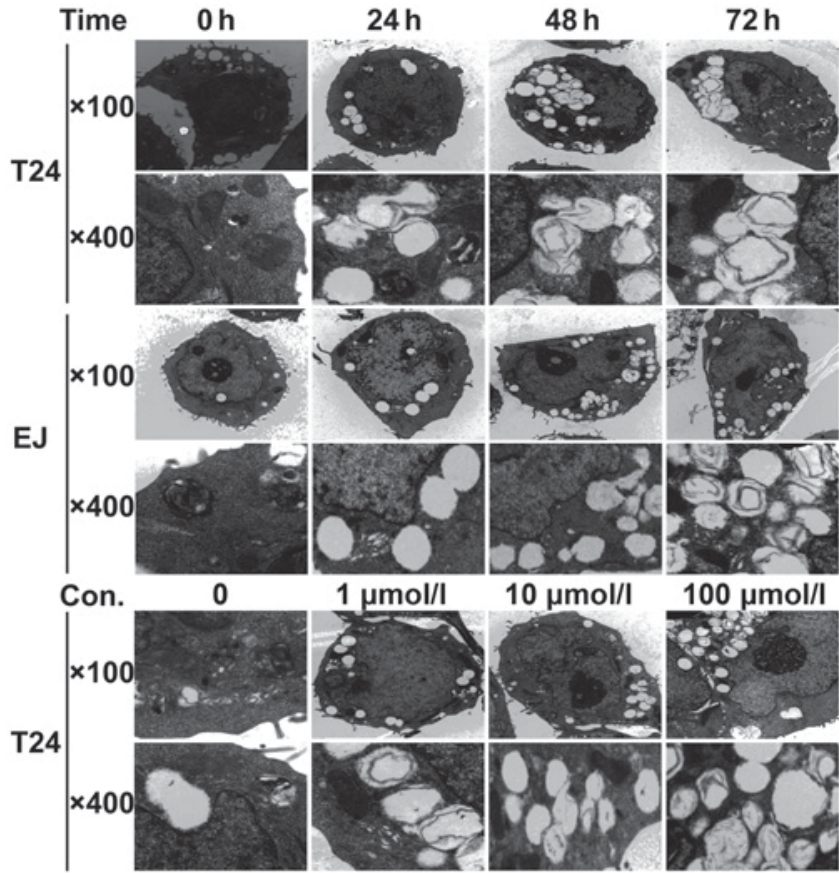

Figure 3. The ultrastructural morphological changes of T24 and EJ cells treated with puerarin, under a transmission electron microscope. Original magnification, $\mathrm{x} 100$.

was performed to confirm the expression levels of associated proteins. As is presented in Fig. 6, compared with the 

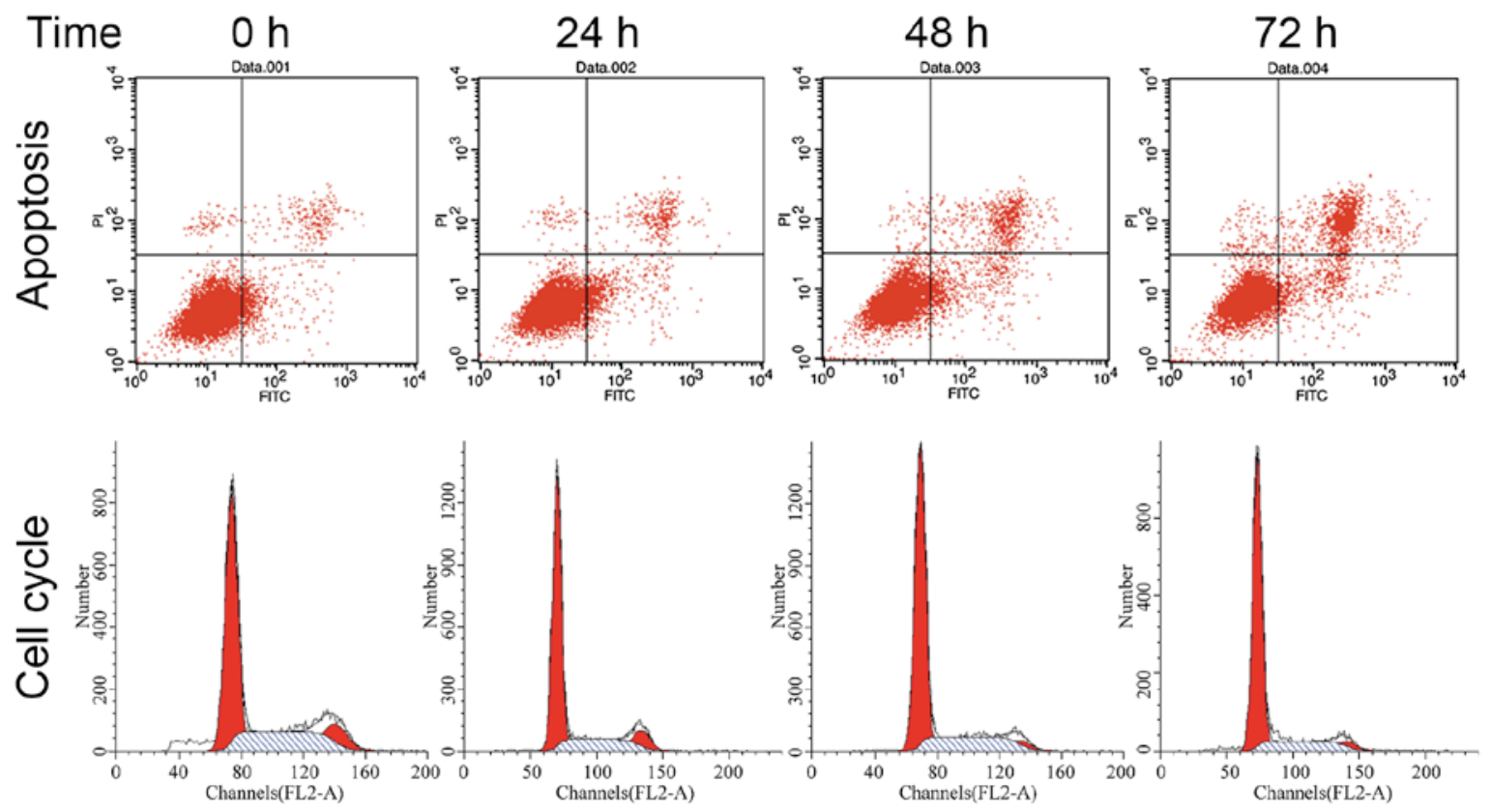

Figure 4. Puerarin affected the cell cycle and apoptosis of T24 cells. T24 cells were treated with puerarin (100 $\mu \mathrm{mol} / 1)$ for 24,48 and $72 \mathrm{~h}$, and then harvested. The cell cycle was analyzed by flow cytometry, and the apoptotic fraction of cells was detected by Annexin V staining following puerarin treatments. FITC, fluorescein isothiocyanate; PI, propidium iodide.
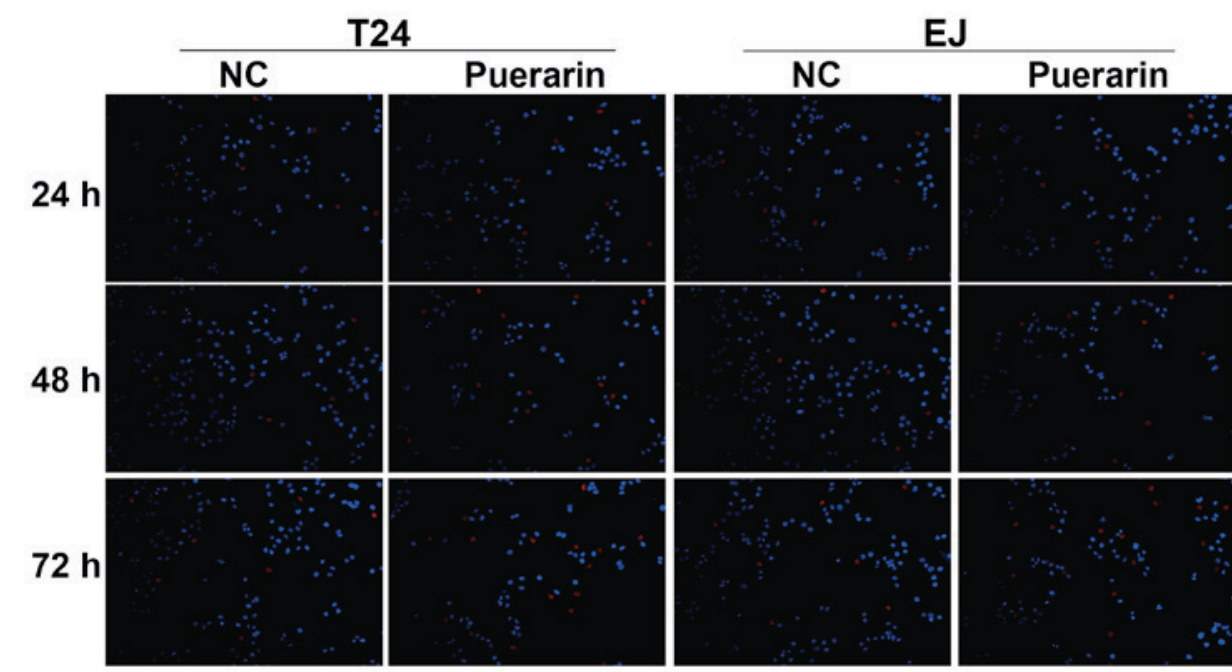

Figure 5. Puerarin induced apoptosis in bladder cancer T24 and EJ cells. T24 and EJ cells were treated with puerarin (100 $\mu$ mol/1) for 24,48 and $72 \mathrm{~h}$, and then harvested. Apoptotic properties were detected by TUNEL assay. Magnification: x100. NC, negative control.

control group, the protein expression level of p-mTOR and p-p70S6K decreased in a dose-dependent manner following puerarin-treatment in T24 and EJ cells, while no changes were observed in the expression level of mTOR and p70S6K in puerarin-treated T24 and EJ cells. In addition, the level of p-mTOR and p-p70S6K decreased in a time-dependent manner following treatment of T24 cells with puerarin, while no changes were observed in the expression level of mTOR and p70S6K in puerarin-treated T24 cells (Fig. 6).

\section{Discussion}

The search for alternative anticancer agents has led to renewed interest in traditional medicine $(25,26)$. Puerarin, as a traditional
Chinese medicine, has protective effects on the nervous and cardiovascular system and may additionally prevent osteoporosis, inflammation and liver injury $(14,15,17)$. Multiple studies have revealed that puerarin induces cell apoptosis, suppresses cell proliferation and increases the chemosensitivity of cancer cells (19-21). Thus, in the present study, a detailed investigation was conducted on the effects of puerarin on bladder cancer cells. As revealed by CCK-8 assay, the viability of T24 and EJ cells was inhibited by puerarin in a dose- and time-dependent manner. The morphological changes induced by puerarin indicated that cells were undergoing apoptosis. With the TUNEL assay, puerarin treatment was revealed to significantly promote the proportion of apoptotic cells. Puerarin may induce bladder cancer cell autophagy and induce cell cycle arrest at the G0/G1 
A

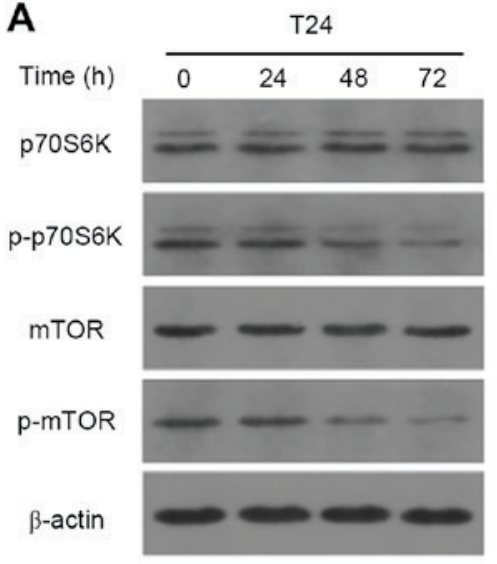

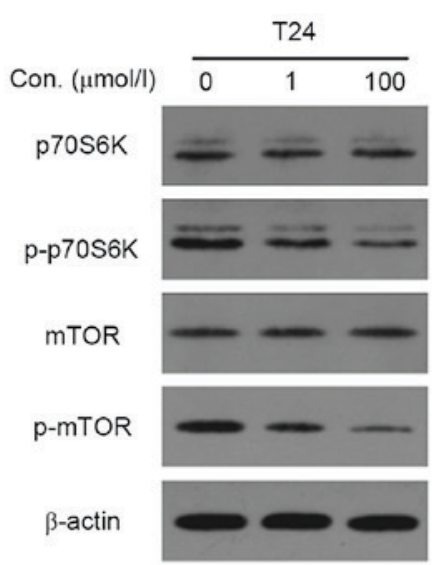

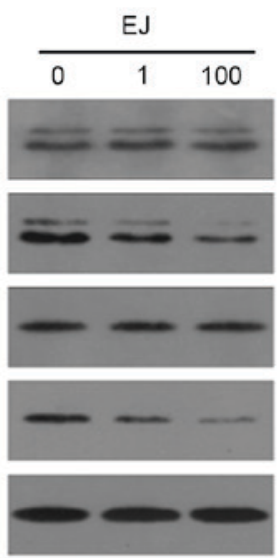

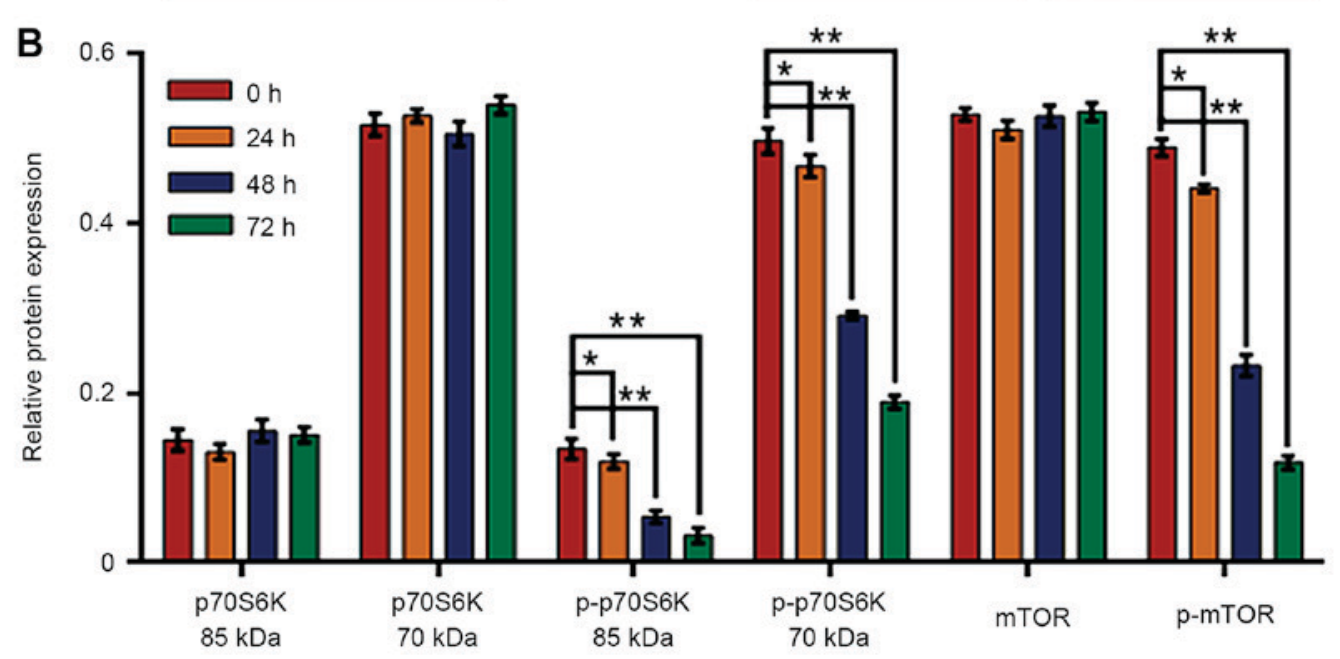

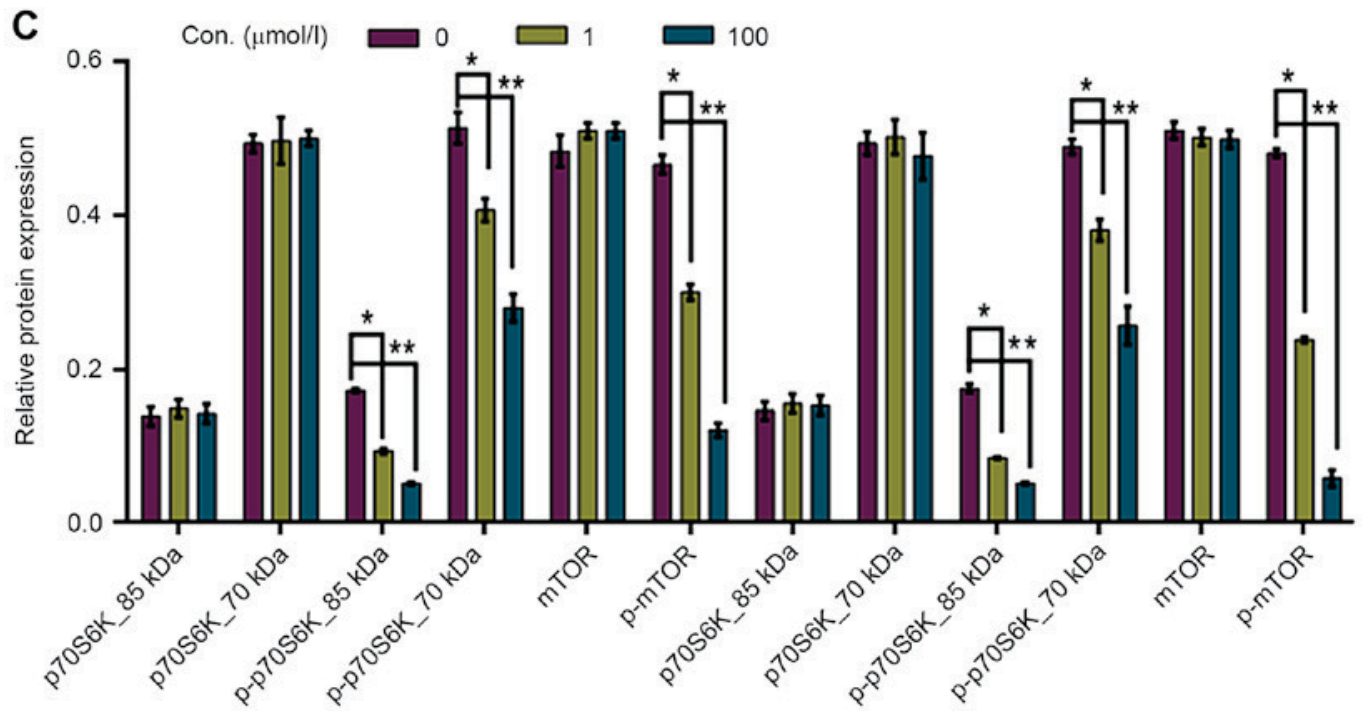

Figure 6. Effect of puerarin on the expression levels of mTOR/p70S6K signaling pathway proteins in T24 and EJ cell lines. (A) Protein levels of mTOR, p70S6K, p-mTOR and p-p70S6K in T24 and EJ cells following puerarin treatment were detected by western blot analysis; (B) Relative protein expression following puerarin treatment in T24 cells for $0,24,48$ and $72 \mathrm{~h} .{ }^{*} \mathrm{P}<0.05,{ }^{* *} \mathrm{P}<0.01 \mathrm{vs} .0 \mathrm{~h}$. (C) Relative protein expression following puerarin treatment in T24 and EJ cells at 0,1 and $100 \mu \mathrm{mol} / 1$. ${ }^{*} \mathrm{P}<0.05,{ }^{* *} \mathrm{P}<0.01 \mathrm{vs} .0 \mu \mathrm{mol} / 1$. mTOR, mechanistic target of rapamycin signaling; p-mTOR, phospho-mTOR; p70S6K, p70-S6 kinase; p-p70S6K, phosphor-P70S6K.

phase. In addition, Transwell assays revealed that puerarin may inhibit cell invasion. These results confirmed that puerarin treatment resulted in an inhibitory effect on bladder cancer cells.

The mTOR signaling pathway is involved in cancer pathogenesis and progression. p70S6K, which is downstream of the mTOR signaling pathway, is associated with tumor formation $(23,27)$. Loss of p70S6K promotes cell cycle progression and cell proliferation (27). p70S6K also mediates the effects of oncogenic protein kinase B (Akt) signaling on mRNA translation, cell growth and tumor progression (28). The activation of the mTOR/S6K axis stimulates protein 
synthesis and cell growth (29). Notably, the mTOR signaling pathway is associated with regulation of energy balance and autophagy as a survival pathway (24). Autophagy is a major degradation pathway in eukaryotic cells, which is essential for removing damaged organelles and macromolecules from the cytoplasm and recycling amino acids during periods of starvation $(30,31)$. Therefore, the present study investigated whether inhibition of the mTOR signaling pathway by puerarin leads to the induction of autophagy. The results indicated that the protein expression levels of p-mTOR and p-p70S6K decreased in a time-dependent manner following treatment of bladder cancer cells with puerarin, and that no changes were observed in the protein expression level of mTOR and p70S6K following puerarin treatment in bladder cancer cells.

$\mathrm{Yu}$ and $\mathrm{Li}$ (21) reported that puerarin induces apoptosis in colon cancer HT-29 cells and suppressing cell proliferation, and puerarin treatment increases the expression level of BCL2-associated X protein (Bax) and decreases the expression level of c-myc and B cell lymphoma-2 (Bcl-2) (21). Yang et al (19) also reported that puerarin exerts antitumor effects through suppressing the expression of p-Akt and $\mathrm{Bcl}-2$, and promotes the expression of Bax in glioblastoma cells. Puerarin inhibited proliferation and induced apoptosis in SMMC-7721 hepatocellular carcinoma cells via the mitochondria-dependent pathway (32). In addition, combined with or without 5-fluorouracil, puerarin significantly suppressed proliferation and markedly increased apoptosis in esophageal cancer cells in vitro and in vivo (18). These previous studies demonstrated similar results to the present study, and thus strengthen them.

A number of previous studies have demonstrated that cell cycle arrest is associated with the inhibition of cancer cell proliferation. For example, Lin et al (33) demonstrated that puerarin inhibits the growth of breast cancer cells through inducing apoptosis and cell cycle arrest in the G2/M phase. Although the results of the present study may differ from those of Lin et al (33), Gan and Yin (34) reported that puerarin treatment leads to cell proliferation inhibition via inducing mantle cell lymphoma cell cycle arrest in the G1 phase, and this mechanism may involve the phosphoinositide-3 kinase/Akt and nuclear factor- $\kappa \mathrm{B}$ signaling pathway. In accordance with this previous study, the results of the present study revealed that puerarin induces bladder cancer cell cycle arrest at the G0/G1 phase. Due to the aforementioned results, it may be concluded that puerarin affects bladder cancer cell viability and induces apoptosis, which is mediated by the mTOR/p70S6K signaling pathway.

Although puerarin has already been widely applied in experimental research and clinical trials in China with high efficiency $(14,15,17,35,36)$, limitations exist. The low aqueous solubility and intestinal permeability values may lead to a lower blood concentration following oral administration of puerarin (37). In order to acquire improved therapeutic effects of puerarin, investigators are attempting to design nanoparticles or other puerarin encapsulations and delivery systems to improve the effect of treatment (38).

In conclusion, the present study demonstrated that puerarin-induced apoptosis in human bladder cancer cells was mediated by activation of the mTOR/p70S6K signaling pathway. Puerarin may serve as a novel therapeutic strategy in the inhibition of carcinogenesis and progression of bladder cancer. However, additional studies are required to affirm the effect of puerarin on bladder cancer in vivo and to verify whether puerarin may be used as part of an intravesical treatment.

\section{Acknowledgements}

The present study was funded by the Hubei Province Health and Family Planning Scientific Research Project (grant no. WJ2017M257), Natural Science Foundation of Hubei Province of China (grant nos. 2014CFC1068 and 2017CFB516) and the Science and Technology Project of Enshi of China (grant no. 2013, 2014).

\section{References}

1. Siegel RL, Miller KD and Jemal A: Cancer statistics, 2015. CA Cancer J Clin 65: 5-29, 2015.

2. Babjuk M, Burger M, Zigeuner R, Shariat SF, van Rhijn BW, Compérat E, Sylvester RJ, Kaasinen E, Böhle A, Palou Redorta J, et al: EAU guidelines on non-muscle-invasive urothelial carcinoma of the bladder: Update 2013. Eur Urol 64: 639-653, 2013.

3. Burger M, Catto JW, Dalbagni G, Grossman HB, Herr H, Karakiewicz P, Kassouf W, Kiemeney LA, La Vecchia C, Shariat S and Lotan Y: Epidemiology and risk factors of urothelial bladder cancer. Eur Urol 63: 234-241, 2013.

4. Schenk-Braat EA and Bangma CH: Immunotherapy for superficial bladder cancer. Cancer Immunol Immunother 54: 414-423, 2005.

5. Shen Z, Shen T, Wientjes MG, O'Donnell MA and Au JL: Intravesical treatments of bladder cancer: Review. Pharm Res 25: 1500-1510, 2008.

6. Bosschieter J, Nieuwenhuijzen JA, van Ginkel T, Vis AN, Witte B, Newling D, Beckers GMA and Moorselaar RJAV: Value of an immediate intravesical instillation of mitomycin $C$ in patients with non-muscle-invasive bladder cancer: A prospective multicentre randomised study in 2243 patients. Eur Urol: July 10, 2017 (Epub ahead of print).

7. Deng T, Liu B, Duan X, Zhang T, Cai C and Zeng G: Systematic review and cumulative analysis of the combination of mitomycin C plus Bacillus Calmette-Guérin (BCG) for non-muscle-invasive bladder cancer. Sci Rep 7: 3172, 2017.

8. Hadaschik BA, ter Borg MG, Jackson J, Sowery RD, So AI, Burt HM and Gleave ME: Paclitaxel and cisplatin as intravesical agents against non-muscle-invasive bladder cancer. BJU Int 101: $1347-1355,2008$.

9. Jiang SJ, Ye LY and Meng FH: Comparison of intravesical bacillus Calmette-Guerin and mitomycin $\mathrm{C}$ administration for non-muscle invasive bladder cancer: A meta-analysis and systematic review. Oncol Lett 11: 2751-2756, 2016.

10. Basmadjian C, Zhao Q, Bentouhami E, Djehal A, Nebigil CG, Johnson RA, Serova M, de Gramont A, Faivre S, Raymond E and Désaubry LG: Cancer wars: Natural products strike back. Front Chem 2: 20, 2014.

11. Orang-Ojong BB, Munyangaju JE, Wei MS, Lin M, Wei FG, Foukunang $\mathrm{C}$ and $\mathrm{Zhu} \mathrm{Y}$ : Impact of natural resources and research on cancer treatment and prevention: A perspective from Cameroon. Mol Clin Oncol 1: 610-620, 2013

12. Keung WM and Vallee BL: Kudzu root: An ancient Chinese source of modern antidipsotropic agents. Phytochemistry 47: 499-506, 1998.

13. Kim MH, Kim SH and Yang WM: Mechanisms of action of phytochemicals from medicinal herbs in the treatment of Alzheimer's disease. Planta Med 80: 1249-1258, 2014.

14. Zhang X, Xiong J, Liu S, Wang L, Huang J, Liu L, Yang J, Zhang G, Guo K, Zhang Z, et al: Puerarin protects dopaminergic neurons in Parkinson's disease models. Neuroscience 280: 88-98, 2014.

15. Tan Y, Liu M and Wu B: Puerarin for acute ischaemic stroke. Cochrane Database Syst Rev 23: CD004955, 2008.

16. Wang Q, Wu T, Chen XY, Duan X, Zheng J, Qiao J, Zhou L, Wei J and Ni J: WITHDRAWN: Puerarin injection for unstable angina pectoris. Cochrane Database Syst Rev: CD004196, 2016. 
17. Liu LJ, Liu LQ, Bo T, Li SJ, Zhu Z, Cui RR and Mao DA: Puerarin suppress apoptosis of human osteoblasts via ERK signaling pathway. Int J Endocrinol 2013: 786574, 2013.

18. Wang J, Yang ZR, Guo XF, Song J, Zhang JX, Wang J and Dong WG: Synergistic effects of puerarin combined with 5-fluorouracil on esophageal cancer. Mol Med Rep 10: 2535-2541, 2014.

19. Yang JA, Li JQ, Shao LM, Yang Q, Liu BH, Wu TF, Wu P, Yi W and Chen QX: Puerarin inhibits proliferation and induces apoptosis in human glioblastoma cell lines. Int J Clin Exp Med 8: 10132-10142, 2015.

20. Guo XF, Yang ZR, Wang J, Lei XF, Lv XG and Dong WG: Synergistic antitumor effect of puerarin combined with 5-fluorouracil on gastric carcinoma. Mol Med Rep 11: 2562-2568, 2015.

21. $\mathrm{Yu} \mathrm{Z}$ and $\mathrm{Li} \mathrm{W}$ : Induction of apoptosis by puerarin in colon cancer HT-29 cells. Cancer Lett 238: 53-60, 2006.

22. O'Toole CM, Povey S, Hepburn P and Franks LM: Identity of some human bladder cancer cell lines. Nature 301: 429-430, 1983.

23. Ching CB and Hansel DE: Expanding therapeutic targets in bladder cancer: The PI3K/Akt/mTOR pathway. Lab Invest 90: 1406-1414, 2010

24. Chen M, Gu J, Delclos GL, Killary AM, Fan Z, Hildebrandt MA, Chamberlain RM, Grossman HB, Dinney CP and Wu X: Genetic variations of the PI3K-AKT-mTOR pathway and clinical outcome in muscle invasive and metastatic bladder cancer patients. Carcinogenesis 31: 1387-1391, 2010.

25. To KK, Au-Yeung SC and Ho YP: Differential nephrotoxicity of cisplatin and a novel series of traditional Chinese medicine-platinum anticancer agents correlates with their chemical reactivity towards sulfur-containing nucleophiles. Anticancer Drugs 17: 673-683, 2006.

26. Mehta RG, Murillo G, Naithani R and Peng X: Cancer chemoprevention by natural products: How far have we come? Pharm Res 27: 950-961, 2010.

27. Chi BH, Kim SJ, Seo HK, Seo HH, Lee SJ, Kwon JK, Lee TJ and Chang IH: P70S6K and Elf4E dual inhibition is essential to control bladder tumor growth and progression in orthotopic mouse non-muscle invasive bladder tumor model. J Korean Med Sci 30: 308-316, 2015.

28. Nawroth R, Stellwagen F, Schulz WA, Stoehr R, Hartmann A, Krause BJ, Gschwend JE and Retz M: S6K1 and 4E-BP1 are independent regulated and control cellular growth in bladder cancer. PLoS One 6: e27509, 2011.
29. Tavares MR, Pavan IC, Amaral CL, Meneguello L, Luchessi AD and Simabuco FM: The S6K protein family in health and disease. Life Sci 131: 1-10, 2015.

30. Arroyo DS, Gaviglio EA, Peralta Ramos JM, Bussi C, Rodriguez-Galan MC and Iribarren P: Autophagy in inflammation, infection, neurodegeneration and cancer. Int Immunopharmacol 18: 55-65, 2014.

31. Mizushima N, Levine B, Cuervo AM and Klionsky DJ: Autophagy fights disease through cellular self-digestion. Nature 451: 1069-1075, 2008.

32. Zhang WG, Liu XF, Meng KW and Hu SY: Puerarin inhibits growth and induces apoptosis in SMMC-7721 hepatocellular carcinoma cells. Mol Med Rep 10: 2752-2758, 2014.

33. Lin YJ, Hou YC, Lin CH, Hsu YA, Sheu JJ, Lai CH, Chen BH, Lee Chao PD, Wan L and Tsai FJ: Puerariae radix isoflavones and their metabolites inhibit growth and induce apoptosis in breast cancer cells. Biochem Biophys Res Commun 378: 683-688, 2009

34. Gan M and Yin X: Puerarin induced in mantle cell lymphoma apoptosis and its possible mechanisms involving multi-signaling pathway. Cell Biochem Biophys 71: 367-373, 2015.

35. Gao Z, Wei B and Qian C: Puerarin injection for treatment of unstable angina pectoris: A meta-analysis and systematic review. Int J Clin Exp Med 8: 14577-14594, 2015.

36. Liang $\mathrm{F}$ and Xie S: Puerarin prevents tumor necrosis factor- $\alpha$-induced apoptosis of PC12 cells via activation of the PI3K/Akt signaling pathway. Exp Ther Med 14: 813-818, 2017.

37. Li H, Dong L, Liu Y, Wang G, Wang G and Qiao Y: Biopharmaceutics classification of puerarin and comparison of perfusion approaches in rats. Int J Pharm 466: 133-138, 2014

38. Liu X, Ding Y, Zhao B, Liu Y, Luo S, Wu J, Li J and Xiang D: In vitro and in vivo evaluation of puerarin-loaded PEGylated mesoporous silica nanoparticles. Drug Dev Ind Pharm 42: 2031-2037, 2016

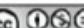
International (CC BY-NC-ND 4.0) License. 